\title{
Benefits of Advanced Full-Wave Vector Analysis Codes for the Design of High-Power Microwave Tubes
}

\author{
A. Marek ${ }^{1}$, K. A. Avramidis ${ }^{1}$, N. S. Ginzburg ${ }^{2}$, S. Illy ${ }^{1}$, J. Jelonnek ${ }^{1,3}$, J. Jin'1 , S. V. Mishakin ${ }^{2}$ and M. Thumm ${ }^{1,3}$ \\ ${ }^{1}$ IHM, ${ }^{3}$ IHE, Karlsruhe Institute of Technology (KIT), Karlsruhe, Germany, alexander.marek@kit.edu \\ ${ }^{2}$ Institute of Applied Physics, Russian Academy of Sciences (IAP- RAS), Nizhny Novgorod, Russia
}

\begin{abstract}
For the design of microwave tubes, precise electromagnetic simulations of the cold structures are of particular importance. In this paper, we present the importance of fullwave vector simulations for the analysis of components in microwave tubes, particularly in high-power gyro-devices. Fullwave vector simulations based on surface integral equations allow an accurate analysis of various structures. A fast solution of surface integral equations is provided by the recently developed advanced acceleration techniques. Simulations of quasi-optical mode converters in high-power gyrotrons, calculations of dispersion relations of gyrotron traveling-wave tubes with helical traveling wave structure and simulations of mirror systems are shown as examples for the wide range of applications of full wave vector simulations in the development of microwave tubes.
\end{abstract}

\section{INTRODUCTION}

$\mathbf{F}$ OR the development of many components in microwave tubes, electromagnetic simulations are of essential importance. Simulation tools are required, which allow an accurate and fast simulation of the electromagnetic field. An advanced simulation tool (KarLESSS) [1] for the simulation of the electromagnetic field scattered by perfect electric conductors of arbitrary shape is under development at the Institute for Pulsed Power and Microwave Technology (IHM) at Karlsruhe Institute of Technology (KIT). The program was developed originally for the analysis of quasi-optical mode converters used in high-power gyrotrons. Those mode converters are electrically large $\left(O\left(10^{4} \lambda^{2}\right)\right)$ and therefore highly optimized tools are required for simulations within a reasonable amount of time. At the same time, the simulations have to be precise. At an output power in the MW range even small uncertainties could have large influence. Besides the analysis of quasioptical mode converters, full-wave vector analysis can be used as accurate simulation tool for various components of highpower microwave tubes. The developed program has been used successfully for the analysis of dispersion relations of gyrotron traveling-wave tubes (gyro-TWTs) with helical interaction circuit [2], the simulation of mirror systems and the synthesis of waveguide mode converters.

The paper is organized as follows. In Section II, a brief overview of the basic algorithms is given. In Section III, examples for simulations of quasi-optical mode converters, dispersion relations of helical traveling wave structures and mirror systems are discussed. Conclusions and suggestions for further work are given in Section IV.

\section{THEORY}

Fast and accurate simulations of electromagnetic fields scattered by arbitrary perfect electric conductors can be reached by full wave simulation methods based on surface integral equations (SIE) such as the electric field integral equation (EFIE) [3]. For the sake of completeness, a short overview of the EFIE and the utilized algorithms to solve the equation is given in this section.

\section{A. EFIE and MoM}

Assuming perfect electrical conductors, the scattering problem can be described with the EFIE [4]:

$$
\begin{aligned}
& -\frac{\mathrm{i}}{\omega \mu} \vec{n}(\vec{r}) \times \vec{E}^{i}(\vec{r})= \\
& \quad \vec{n}(\vec{r}) \times \iint_{S} \mathrm{~d} \overrightarrow{r^{\prime}} G\left(\vec{r}, \overrightarrow{r^{\prime}}\right)\left[\vec{J}\left(\overrightarrow{r^{\prime}}\right)+\frac{1}{k^{2}} \nabla^{\prime} \nabla^{\prime} \cdot \vec{J}\left(\overrightarrow{r^{\prime}}\right)\right]
\end{aligned}
$$

where $\vec{J}$ represents the unknown electrical surface current, $\vec{E}^{i}$ is the known incident field and $G$ is the free space Green's function. In our implementation, the EFIE is solved numerically with a Galerkin-type method of moments (MoM) [5]. In the MoM the currents are expanded into a sum of weighted basis functions $\vec{f}$. Inserting the expansion to (1), making use of the linearity of the equation and taking an inner product $(\langle\cdot, \cdot\rangle)$ of the equation with the basis functions, the problem can be formulated as a matrix equation. For the lowest order basis functions only one unknown for every basis function appears and the matrix equation gets the following form:

$$
\left\langle\vec{f}_{m}, \vec{E}^{i}\right\rangle=\sum_{n=1}^{N} a_{n}\left\langle\vec{f}_{m}, L\left(\vec{f}_{n}\right)\right\rangle
$$

where $L$ represents the linear operator on the right site of (1). For higher order basis functions, several unknowns appear for every basis function and therefore a set of coupled equations have to be solved. 


\section{B. Basis Functions}

To achieve an optimal accuracy and runtime for various applications, different sets of basis functions such as the RaoWilton-Glisson (RWG) basis functions [6] and higher order basis functions for curved triangles [7] are implemented in KarLESSS. As default for the simulation of quasi-optical systems a set of mixed first order basis functions for curved triangles is used. The first order basis functions allow the reduction of unknowns by round about $30 \%$ compared to the RWG basis functions. The chosen basis functions are suitable for every surface parametrized with $C^{(1,1)}$ continuity. It has been shown that a parametrization with local bicubic B-splines [8] can accurately fit surfaces with small arbitrary perturbations as e.g. in modern quasi-optical mode converters.

\section{Adaptive Cross Approximation}

The Green's function in the EFIE is a non-local operator. Thus, calculating the impedance matrix by the MoM results in a dense matrix. Consequently, the storage requirements for the impedance matrix are of the order $O\left(N^{2}\right)$, where $N$ denotes the number of unknowns. Additionally, the computation costs for the matrix fill-in and for every matrix-vector product, which are needed for the iterative solution of the system of linear equations, are of $O\left(N^{2}\right)$ as well. Therefore, the computational demands for solving the EFIE for highly oversized scatterers such as quasi-optical mode converters of high-power gyrotrons are prohibitive. In the recent years, several solutions have been developed to mitigate this problem. Well known methods are AIM [9], MLFMA [10], MLMDA [11], SVDMDA [12]. In KarLESSS, the adaptive cross approximation algorithm (ACA) has been implemented, which was first used for electromagnetic problems in [13]. An advantage of the ACA is its purely algebraic manner. The premise of the ACA algorithm is that the interactions of well-separated parts of the geometry cause a rank-deficient impedance matrix. Therefore, the impedance matrix of an electrically large problem consists of many numerically rank deficient sub-matrices $Z^{m \times n}$. The ACA performs a rank-revealing LU decomposition of these sub-matrices and compresses them efficiently. Every $Z^{m \times n}$ is approximated by a compressed sub-matrix $\tilde{Z}^{m \times n}$ :

$$
Z^{m \times n} \approx \tilde{Z}^{m \times n}=U^{m \times r} V^{r \times n}
$$

where $r$ is the rank of the approximate matrix $\tilde{Z}$. If $r<\frac{m \cdot n}{m+n}$, the approximated matrix $\tilde{Z}$ requires less memory and can be calculated faster than the exact matrix $Z$. An advantage of the ACA algorithm is its iterative calculation of $\tilde{Z}$ (see [13] for details). Because of this iterative calculation only a partial knowledge of the original matrix is required, which reduces the calculation time as well as the required memory. The ACA algorithm has a complexity of $O\left(N^{3 / 2} \log (N)\right)$ [13]. For electrically large problems with a size of more than 50 wavelengths, the scaling of the ACA algorithm can even decline [14]. To overcome this undesirable behavior, several optimizations have been developed in the last years. We have chosen the so called sparsified ACA (SPACA) [15], where a sampling of the basis functions is combined with a matrix decomposition algorithm and an additional singular value compression (SVD-MDA) [12]. With the SPACA the favorable complexity of $N \log (N)$ is reached for electrically mediumsized problems. But even for electrically large problems, simulations can be performed in a suitable amount of time and memory on modern computers.

For small problems, the resulting system of linear equations can be solved with a direct solver. For electrically large problems an iterative solver is more suitable. In our implementation an iterative solver based on a flexible generalized minimal residual method (FGMRES) [16] is used.

\section{Simulations}

The simulation program is implemented in an objectoriented manner in the programming language $\mathrm{C}++$. For algebraic operations the library Armadillo [17] is used. The implementation provides a high flexibility and the possibility for further developments and optimizations. An advantage of the ACA based algorithms is the easy implementation for parallel computing. Our implementation supports parallelized calculations on multiple cores of shared memory systems as well as on several devices in distributed memory systems.

\section{A. Quasi-Optical System for High Power Gyrotrons}

A critical component in high-power gyrotrons is the quasioptical mode converter. Because of the high energy output of gyrotrons used e.g. in fusion experiments, it is important to design efficient quasi-optical mode converters with low stray radiation and high Gaussian mode content at the output window. Simulation tools based on the EFIE allow a fullwave simulation including all diffraction and reflection effects within a short calculation time and with a high accuracy. In Fig. 1, the simulated field distribution for the quasi-optical system of the $1 \mathrm{MW}$ gyrotron for the stellarator W7-X [18] is shown. The gyrotron operates at a frequency of $140 \mathrm{GHz}$ and in the $\mathrm{TE}_{28,8}$ mode. The quasi-optical system consists of a launcher and three mirrors with a total area of $24700 \lambda^{2}$. For an accurate simulation $6 \cdot 10^{5}$ unknowns are required. Because of the parallelized implementation the simulation can be performed in less than $1 \mathrm{~h}$ on 4 Intel Xeon E5-2667 CPUs. The volume of the whole quasi-optical system is very large. Therefore, simulation methods based on a discretization of the volume, as e.g. the finite element method (FEM) or the finite difference method (FDM), are not applicable to this problem. An alternative to the SIEs are methods based on the scalar Helmholtz equation. These allow a very fast calculation but because of there scalar nature they can not take refraction effects into account.

\section{B. Mirror Systems}

A similar problem to the previous simulation of the quasioptical systems, is the simulation of an input-output system for a gyro-TWT (see Fig. 2 a)). The system is designed for a center frequency of $260 \mathrm{GHz}$ and consists of parabolic mirrors and sinusoidally corrugated mirrors, which are used as polarization separators. Shown is the simulation of the output channel. For the simulation, a linearly polarized $\mathrm{HE}_{1,1}$ beam is radiated 


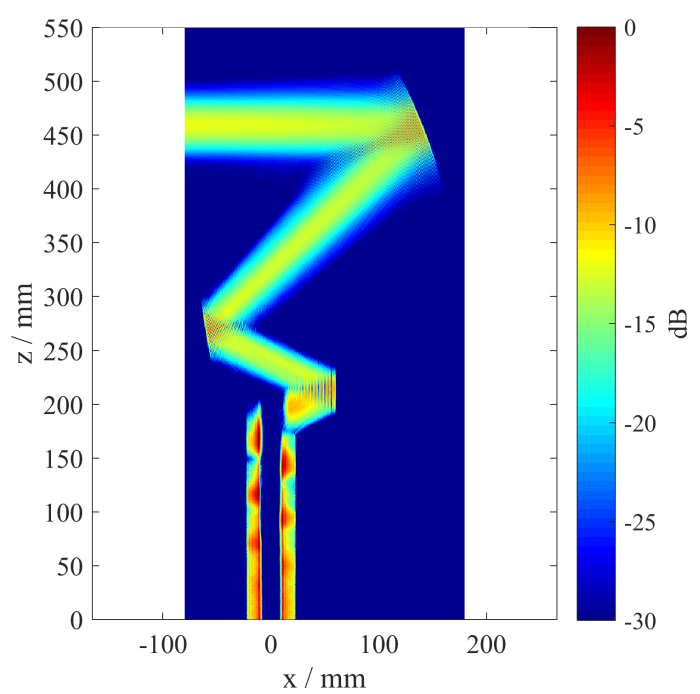

Fig. 1. Simulated distribution of the electric field in the $x-z$ plane of the quasi-optical system of the $140 \mathrm{GHz}, 1 \mathrm{MW}$ gyrotron for W7-X.

from the position of the gyro-TWT. After it is reflected on the mirrors 1, 2 and 3, the field distribution is analyzed at the plane of the output-port. In Fig. 3, the amplitude and the phase at the output-port are shown. In addition to the separate simulation of the input and output channels, a simultaneous simulation of both channels was performed. The resulting surface currents on the mirrors of the output channel are shown in Fig. 2 b). The simulation can be performed in less than 10 minutes. The benefit of the simulation based on SIEs is similar to the previous example. The required computational resources are independent from the volume of the total problem and hereby also independent of the distances between the mirrors.
(1)

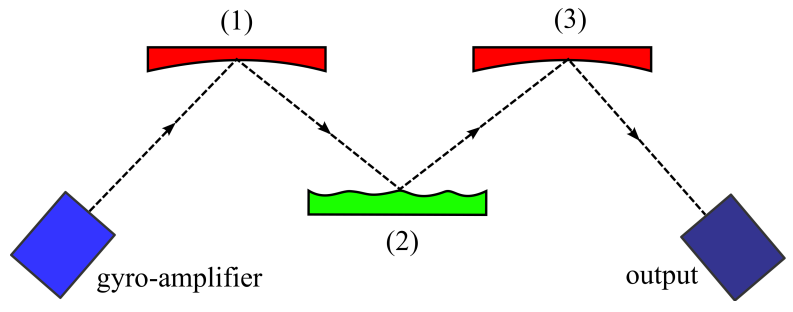

(a)

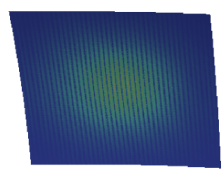

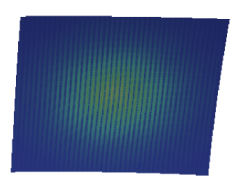

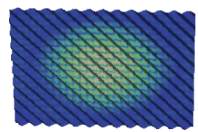

(b)
Fig. 2. Part of a mirror system for a gyro-TWT I/O system. a) Schematic draw. b) Calculated currents on the mirrors (a.u.).

\section{Helically Corrugated Waveguides}

Waveguides with various types of periodic corrugation are widely used in microwave tubes, e.g. for gyro-TWTs. A gyro-
TWT with helically corrugated interaction circuit was first demonstrated in [2]. The profile of the helically corrugated wall is described by:

$$
r(\phi, z)=r_{0}+l \sin \left(\frac{2 \pi}{d} z-3 \phi\right)
$$

where $r_{0}$ defines the main radius of the waveguide, $l$ is the corrugation depth and $d$ is the period length of the corrugation. At the beginning and at the end of the circuit, tapers with a length of a few periods transform the mode of a circular waveguide into eigen modes of the corrugated waveguide. The threefold right-handed corrugation couples the righthanded $\mathrm{TE}_{2,1}$-mode with the forward propagating left-handed $\mathrm{TE}_{-1,1}$-input mode, whereas a right-handed $\mathrm{TE}_{1,1}$-input mode is almost not affected. The dispersion characteristics of such a structure can be described analytically with the method of perturbations [2]. However, this method provides reasonable accuracy only if the corrugation depth is much smaller than the operating wavelength. For structures which do not fulfill this restriction, examples can be found where simulations based on the finite-difference method or the finite-element method are used (see [19] and [20]). In this paper, we propose that simulation tools based on the EFIE can be also used for the calculation of the dispersion relation of the helically corrugated waveguides. The benefit of methods based on the MoM and SIEs is that they are devoid of numerical dispersion and dissipation effects. As an example we calculate the dispersion relation for a helical waveguide design for a gyro-TWT operating at the center frequency of $260 \mathrm{GHz}$. For that, simulations are performed at different frequencies and the radial electric field distribution along a line parallel

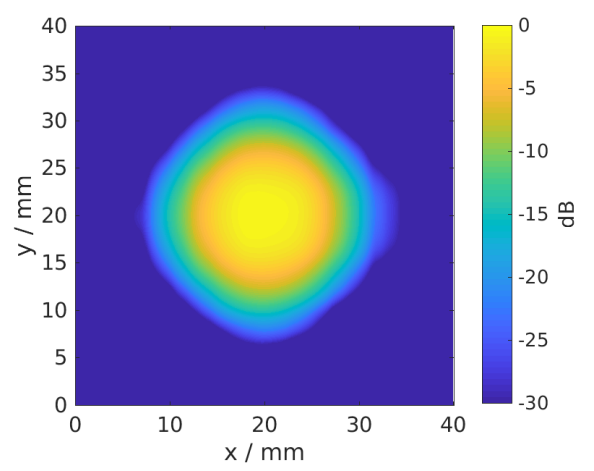

(a)

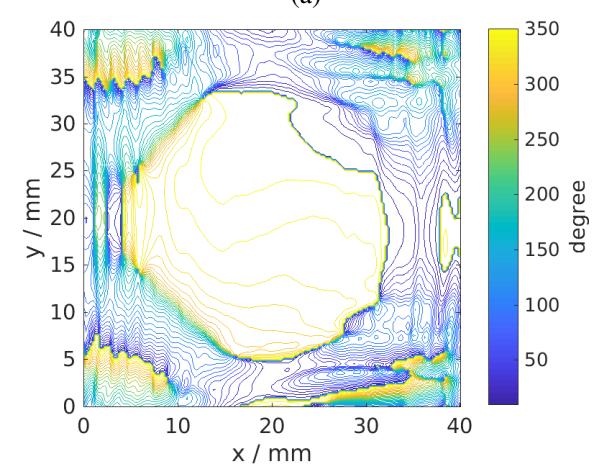

(b)

Fig. 3. Simulated field distribution for a frequency of $260 \mathrm{GHz}$ at the position of the output port. 
to the $z$ axis is taken. With a spatial Fourier transform the axial wavenumber is determined and we observe the dispersion relation shown in Fig. 4. A single calculation can be performed in less than 1 minute.

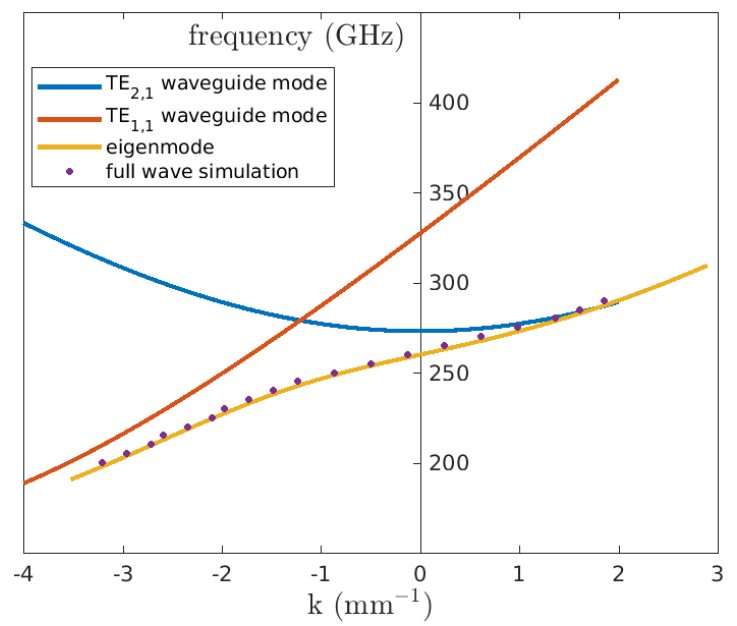

Fig. 4. Dispersion diagram for a helically corrugated waveguide, calculated with a 2D-FEM code [20] and the full-wave simulation tool KarLESSS.

\section{CONCLUSION}

In this paper, possible applications of advanced algorithms for electromagnetic simulations of different components in microwave tubes have been presented. The combination of the SPACA, first order basis functions, curved surfaces parametrized by B-splines and the implementation for parallel computing allows an accurate and fast simulation of the electric fields. The benefits of the presented methods are the full-wave simulation including all refraction effects, the independence of the problem's total volume and the devoid of numerical dispersion and dissipation effects. In future, the capability for simulations of other microwave components e.g. miter bends and waveguide mode converters will be considered. In particular, the developed simulation tool will be used for numeric optimizations of various components based on genetic algorithms and similar optimization methods.

\section{ACKNOWLEDGMENT}

The research is supported by the joint RSF-DFG project (Je 711/1-1) Generation of Ultrashort Pulses in Millimeter and Submillimeter Bands for Spectroscopy and Diagnostic of Various Media Based on Passive Mode-locking in Electronic Devices with Nonlinear Cyclotron Absorber in the Feedback Loop.

\section{REFERENCES}

[1] A. Marek, K. Avramidis, S. Copplestone, N. Ginzburg, S. Illy, J. Jelonnek, J. Jin, S. Mishakin, A.-S. Müller, P. Ortwein et al., "Simulation of electromagnetic fields scattered from arbitrary shaped electric conductors," in EPJ Web of Conferences, vol. 149. EDP Sciences, 2017, p. 04016.

[2] G. G. Denisov, V. L. Bratman, A. D. R. Phelps, and S. V. Samsonov, "Gyro-twt with a helical operating waveguide: new possibilities to enhance efficiency and frequency bandwidth," IEEE Transactions on Plasma Science, vol. 26, no. 3, pp. 508-518, Jun 1998.
[3] S. Sabchevski, I. Zhelyazkov, E. Benova, V. Atanassov, P. Dankov, M. Thumm, A. Arnold, J. Jin, and T. Rzesnicki, "Quasi-optical converters for high-power gyrotrons: a brief review of physical models, numerical methods and computer codes," Journal of Physics: Conference Series, vol. 44, no. 1, p. 102, 2006.

[4] W. C. Gibson, The method of moments in electromagnetics. CRC press, 2007.

[5] M. M. Ney, "Method of moments as applied to electromagnetic problems," IEEE Transactions on Microwave Theory and Techniques, vol. 33, no. 10, pp. 972-980, Oct 1985

[6] S. M. Rao, D. R. Wilton, and A. W. Glisson, "Electromagnetic scattering by surfaces of arbitrary shape," IEEE Transactions on Antennas and Propagation, vol. AP-30, pp. 409-418, 1982.

[7] W. Cai, "High-Order Mixed Current Basis Functions for Electromagnetic Scattering of Curved Surfaces." J. Sci. Comput., vol. 14, no. 1, pp. 73105, 1999.

[8] L. A. Piegl and W. Tiller, The NURBS book (2. ed.)., ser. Monographs in visual communication. Springer, 1997.

[9] E. Bleszynski, M. Bleszynski, and T. Jaroszewicz, "Aim: Adaptive integral method for solving large-scale electromagnetic scattering and radiation problems," Radio Science, vol. 31, no. 5, pp. 1225-1251, 1996.

[10] J. Song, C.-C. Lu, and W. C. Chew, "Multilevel fast multipole algorithm for electromagnetic scattering by large complex objects," IEEE Transactions on Antennas and Propagation, vol. 45, no. 10, pp. 1488-1493, 1997.

[11] E. Michielssen and A. Boag, "A multilevel matrix decomposition algorithm for analyzing scattering from large structures," IEEE Transactions on Antennas and Propagation, vol. 44, no. 8, pp. 1086-1093, 1996.

[12] J. M. Rius, J. Parron, A. Heldring, J. M. Tamayo, and E. Ubeda, "Fast iterative solution of integral equations with method of moments and matrix decomposition algorithm-singular value decomposition," IEEE Transactions on Antennas and Propagation, vol. 56, no. 8, pp. 23142324, 2008

[13] K. Zhao, M. N. Vouvakis, and J.-F. Lee, "The adaptive cross approximation algorithm for accelerated method of moments computations of emc problems," IEEE Transactions on Electromagnetic Compatibility, vol. 47 , no. 4, pp. 763-773, 2005.

[14] A. Heldring, J. Tamayo, and J. Rius, "On the degrees of freedom in the interaction between sets of elementary scatterers," in Antennas and Propagation, 2009. EuCAP 2009. 3rd European Conference on. IEEE, 2009, pp. 2511-2514.

[15] A. Heldring, J. M. Tamayo, C. Simon, E. Ubeda, and J. M. Rius, "Sparsified adaptive cross approximation algorithm for accelerated method of moments computations," IEEE Transactions on Antennas and Propagation, vol. 61, no. 1, pp. 240-246, Jan 2013.

[16] Y. Saad, "A flexible inner-outer preconditioned gmres algorithm," SIAM Journal on Scientific Computing, vol. 14, no. 2, pp. 461-469, 1993.

[17] C. Sanderson and R. Curtin, "Armadillo: a template-based c++ library for linear algebra," Journal of Open Source Software, 2016.

[18] G. Gantenbein, V. Erckmann, S. Illy, S. Kern, W. Kasparek, C. Lechte, W. Leonhardt, C. Livin, A. Samartsev, A. Schlaich, M. Schmid, and M. Thumm, "140 ghz, $1 \mathrm{mw} \mathrm{cw}$ gyrotron development for the ech system of the stellarator w7-x," in 35th International Conference on Infrared, Millimeter, and Terahertz Waves, Sept 2010, pp. 1-3.

[19] G. Burt, S. Samsonov, K. Ronald, G. Denisov, A. Young, V. Bratman, A. Phelps, A. Cross, I. Konoplev, W. He et al., "Dispersion of helically corrugated waveguides: Analytical, numerical, and experimental study," Physical review E, vol. 70, no. 4, p. 046402, 2004.

[20] S. V. Mishakin and S. V. Samsonov, "Analysis of dispersion and losses in helically corrugated metallic waveguides by 2 -d vector finite-element method," IEEE Transactions on Microwave Theory and Techniques, vol. 59, no. 9, pp. 2189-2196, 2011. 